Journal for ImmunoTherapy of Cancer

\title{
CD103+ tumor-resident CD8+ $T$ cell numbers underlie improved patient survival in oropharyngeal squamous cell carcinoma
}

\author{
Rehana Hewavisenti, ${ }^{1}$ Angela Ferguson, ${ }^{1,2,3}$ Kevin Wang, ${ }^{4}$ Deanna Jones, ${ }^{5}$ \\ Thomas Gebhardt, ${ }^{6}$ Jarem Edwards, ${ }^{1}$ Mei Zhang, ${ }^{5,7}$ Warwick Britton, ${ }^{1}$ Jean Yang, ${ }^{4}$ \\ Angela Hong, ${ }^{5,7}$ Umaimainthan Palendira (i) $1,2,3$
}

To cite: Hewavisenti $\mathrm{R}$, Ferguson $\mathrm{A}$, Wang $\mathrm{K}$, et al. CD103+ tumor-resident CD8+ T cell numbers underlie improved patient survival in oropharyngeal squamous cell carcinoma. Journal for ImmunoTherapy of Cancer 2020;8:e000452. doi:10.1136/jitc-2019-000452

- Additional material is published online only. To view please visit the journal online (http://dx.doi.org/10.1136/jitc2019-000452).

$\mathrm{RH}$ and $\mathrm{AF}$ contributed equally.

AH and UP contributed equally. AH and UP are joint senior authors.

Accepted 21 April 2020

Check for updates

(c) Author(s) (or their employer(s)) 2020. Re-use permitted under CC BY-NC. No commercial re-use. See rights and permissions. Published by BMJ.

For numbered affiliations see end of article.

\section{Correspondence to}

Dr Umaimainthan Palendira; umaimainthan.palendira@ sydney.edu.au

Professor Angela Hong; angela.hong@sydney.edu.au

\section{ABSTRACT}

Background Human Papillomavirus (HPV) associated oropharyngeal squamous cell carcinoma (OPSCC) is one of the fastest growing cancers in the Western world. When compared to OPSCCs induced by smoking or alcohol, patients with HPV+ OPSCC, have better survival and the mechanisms remain unclear.

Methods The Cancer Genome Atlas (TCGA) database was examined for genes associated with tissue-resident CD8+ T cells. Multiplex immunohistochemistry $(\mathrm{HC})$ staining was performed on tumor specimen taken from $35 \mathrm{HPV}+$ and 27 HPV- OPSCC patients.

Results TCGA database revealed that the expression of genes encoding CD103 and CD69 were significantly higher in HPV+ head and neck SCCs (HNSCC) than in HPV- HNSCC. Higher expression levels of these two genes were also associated with better overall survival. IHC staining showed that the proportion of CD103+ tumorresident CD8+ $\mathrm{T}$ cells were significantly higher in HPV+ OPSCCs when compared to HPV- OPSCC. This higher level was also associated with both lower risk of loco-regional failure, and better overall survival. Importantly, patients with HPV- OPSCC who had comparable levels of CD103+ tumor-resident CD8+ T cells to those with HPV+ OPSCC demonstrated similar survival as those with HPV+OPSCC. Conclusion Our results show that CD103+ tumorresident CD8+ $T$ cells are critical for protective immunity in both types of OPSCCs. Our data further suggest that the enhanced local protective immunity provided by tumorresident $\mathrm{T}$ cell responses is the underlying factor driving favorable clinical outcomes in HPV+ OPSCCs over HPVOPSCCs.

\section{INTRODUCTION}

Oropharyngeal squamous cell carcinomas (OPSCCs) have two distinct etiologies: human papillomavirus (HPV)-induced or smokingrelated. While the incidence of HPV-negative (HPV-) OPSCC has steadily declined over the past few decades, the incidence of HPVpositive (HPV+) OPSCCs has increased to become one of the fastest growing cancers in some Western countries. ${ }^{1-3}$ HPV+ OPSCC is clinically and biologically distinct ${ }^{4-6}$ and accounts for up to $70 \%$ of OPSCCs in the Western world. ${ }^{7}$ Among the many different strains of HPV, type 16 is the most dominant type associated with OPSCC, as observed in cervical cancer $^{8}$ and anogenital cancer. ${ }^{9}$ Patients with HPV+ OPSCC tend to be younger and have more advanced nodal disease at diagnosis but better outcomes. This difference in survival has been attributed to many factors, including cell intrinsic factors such as protection of p53 to immunogenicity of viral proteins inducing better immune responses. ${ }^{10}$ Understanding the immunological mechanisms involved in this survival advantage can help design novel therapeutic strategies.

Tissue-resident memory (Trm) $\mathrm{T}$ cells have recently come under intense focus after several studies showed correlations of their numbers with better patient survival in many different cancers. ${ }^{11}$ Identified as a subset of non-circulating $\mathrm{T}$ cells permanently residing in tissues, these $\mathrm{CD} 103+\mathrm{CD} 8+\mathrm{Trm} \mathrm{T}$ cells were initially implicated in protective immunity against many pathogens, including those that establish persisting or latent infections. ${ }^{12}$ It has become evident that these cells play a crucial role in local immunity against these pathogens by providing the first line of adaptive immune resistance. ${ }^{12}$ We recently showed that these cells were better predictors of survival than the total CD8+ Tcell counts in patients with metastatic melanoma, ${ }^{13}$ with similar findings supported in many other malignancies such as cervical cancer, ${ }^{14}$ ovarian cancer, ${ }^{15}$ breast cancer, ${ }^{16}$ and lung cancer. ${ }^{17}{ }^{18}$ Mechanistically, a recent study has shown that these cells play an important role in preventing tumor metastasis by maintaining a stage of immune equilibrium. ${ }^{19}$ As 
such, it is highly likely that Trm T cells generated against HPV are likely to play a key role in HPV-driven OPSCCs.

In this study, we determined the prognostic significance of Trm T cells in a well-defined cohort of OPSCC by their HPV status. In addition, we examined whether the Trm $\mathrm{T}$ cells can modify the known prognostic effect of HPV in OPSCC.

\section{METHODS \\ Patients}

The study cohort consisted of 62 patients with T1-4 N0-3 M0 (American Joint Committee on Cancer Staging System Seventh Edition) OPSCC treated with curative intent. Demographic characteristics, clinicopathological details, and follow-up data were obtained from institutional databases. The histopathology and tumor grade were reviewed in all cases. The median age was 58 (range 34-83; online supplementary table 1). The median follow-up time was 65.5 months. All samples were either from definitive surgery or biopsy prior to radiotherapy with or withoutchemotherapy.

\section{HPV testing}

We determined HPV status of the OPSCC by both DNA and p16 expression as reported previously. ${ }^{2}{ }^{6}$ The presence and type of HPV DNA were determined by E6-based multiplex tandem PCR assay using a modification of the Tandem method of Stanley and Szewczuk. ${ }^{8}$ This assay simultaneously detects and identifies $21 \mathrm{HPV}$ types. The expression of p16 was determined by semiquantitative immunohistochemistry (IHC) using the JC2 p16 antibody (Neomarkers, Fremont, USA). Staining was typically strong and diffuse in both the nucleus and cytoplasm of cancer cells and was recorded as positive if seen in more than $50 \%$ of cancer cells. ${ }^{9}$ An HPV+ OPSCC was defined as one both testing positive for HPV DNA and demonstrating p16 overexpression by IHC. ${ }^{20}{ }^{21}$

\section{Gene expression analysis from TCGA database}

The expression levels of genes associated with Trm T cells and the impact on patient survival were determined from the publicly available The Cancer Genome Atlas (TCGA) database using recently published Tumor Immune Estimation Resource server. ${ }^{22}$ Differential expression module was used to determine the expression levels of ITGAE (CD103) and CD69 between patients with HPV+ and HPV- head and neck squamous cell carcinoma (HNSCC). Survival module was used to determine the impact of increased gene expression levels on overall patient survival.

\section{Immunohistochemical staining of tissues}

Formalin fixed paraffin embedded (FFPE) OPSCC tissue blocks were cut at $4 \mu \mathrm{m}$ thickness and stained using Opal Multiplex IHC assay kit (PerkinElmer, Waltham, Massachusetts, USA) as per optimized inhouse protocol. Briefly, FFPE tissue sections were deparaffinized and rehydrated.
Tissues were then subjected to antigen retrieval by boiling in basic $(10 \mathrm{mmol} / \mathrm{L}$ Tris base, $1 \mathrm{mmol} / \mathrm{L}$ EDTA, $0.05 \%$ Tween 20, pH 9.0) buffer. Tissue sections were then incubated with $3 \%$ hydrogen peroxide for 30 minutes at room temperature before washing and blocking. Sections were then incubated with a single unconjugated primary antibody for $35 \mathrm{~min}$, washed and then incubated with Opal Polymer HRP (Akoya Biosciences, USA) for 10 minutes. After washing, sections were incubated with opal fluorochromes at a 1:100 dilution made up in tyramide signal amplification reagent (PerkinElmer) for 10 minutes. The antigen retrieval step was repeated for subsequent stains. After all staining was complete, sections were stained with 4', 6-diamidino-2-phenylindole, dihydrochloride (DAPI) (Cell Signaling Technologies) for 5 minutes and then mounted using Vectashield (Vector Labs, USA). Slides are kept in the dark until imaging. The following antibodies were used: Ab4055 clone (Abcam, UK) for investigating CD8 expression and Ab129202 clone (Abcam) for CD103 expression. Mantra imaging platform (PerkinElmer) was used for imaging and Mantra Snap software was used for the acquisition of data. For all quantitative analyses, up to 15 randomly selected regions of interest per section were analyzed. Images were unmixed and analyzed using inForm 2.3.0 software (PerkinElmer) to phenotype and quantify the expression of each of the markers on individual cells.

\section{Statistical analyses}

Graphical and statistical analyses were performed using Prism V.6.0f (GraphPad Software), or survival analysis was performed in the $\mathrm{R}$ software with the survival package (V.2.44) and the survminer package (V.0.4.6). Mann-Whitney rank tests for unpaired, non-parametric distributions were used to identify differences between proportions of immune cell subsets (ns; *p<0.05; $* * \mathrm{p}<0.01 ; * * * \mathrm{p}<0.001$; and $* * * * \mathrm{p}<0.0001)$, or Wilcoxon rank-sum test was applied for comparisons of cell densities in different segments of the tumors. Both overall survival and recurrence-free survival times were censored either at the 120th months after the initial diagnosis or where follow-up ended. The median of each CD8 segmentation was used as the threshold value to separate groups. Variability in the data is shown as SEM. All clinical and genomic variables that potentially impact survival were included in the multivariate analyses of immunemediated survival data.

\section{RESULTS AND DISCUSSION \\ Genes associated with tumor-resident CD8+ $T$ cells are enriched in HPV+ HNSCC and are associated with better prognosis}

We examined TCGA database to determine whether ITGAE (CD103) and CD69, the two genes associated with CD8+ Trm $\mathrm{T}$ cells, were differentially expressed between HPV+ and HPV- HNSCCs. HPV+ HNSCCs had significantly higher expression of both these genes when 

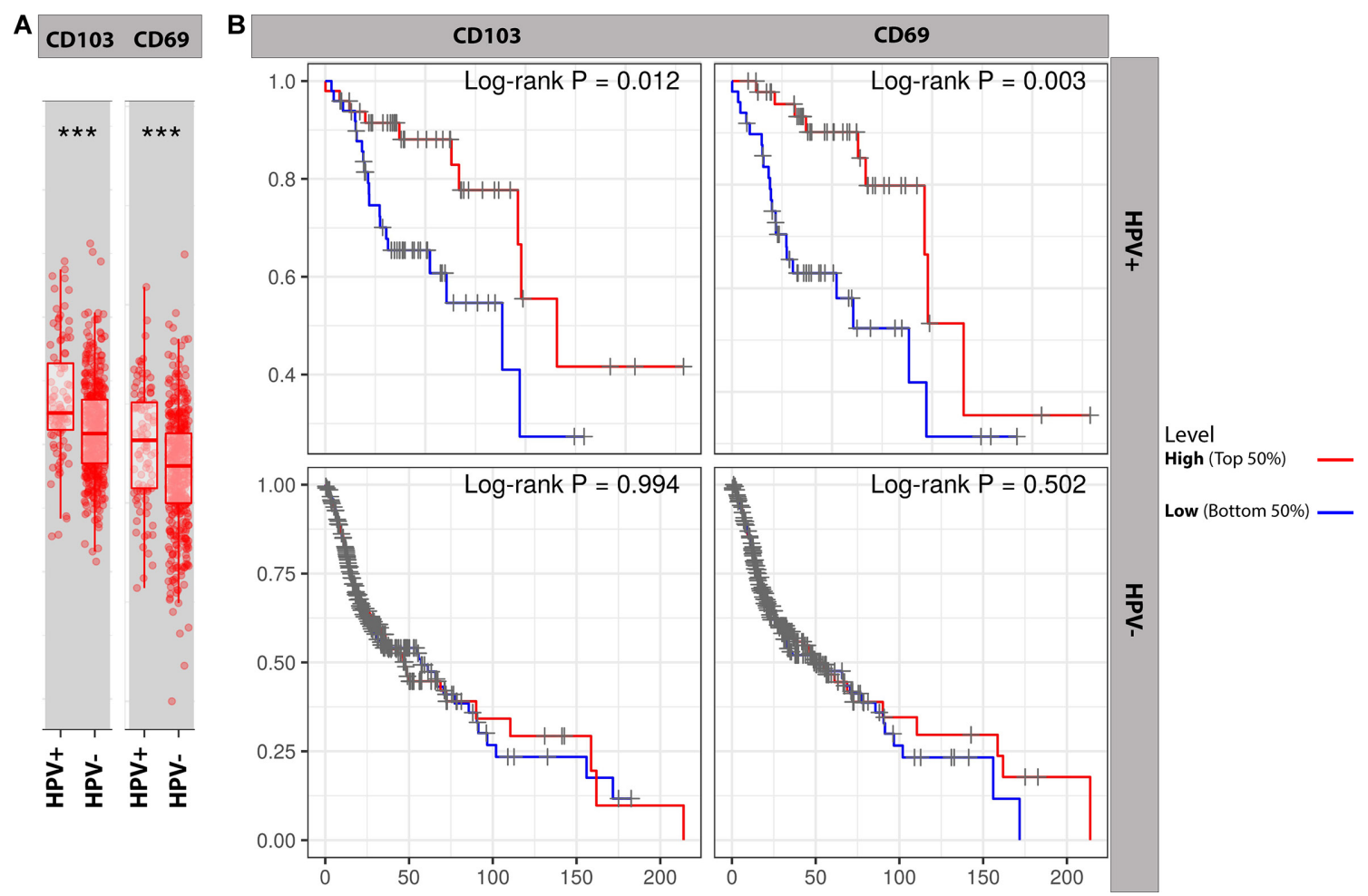

Figure 1 Increased expression of genes related to tissue-resident T cells is associated with improved patient survival in HPV+ head and neck SCC. The expression levels of two genes associated with tissue-resident T cells, ITGAE (CD103) and CD69, were examined in The Cancer Genome Atlas database using the Tumor Immune Estimation Resource server. (A) Differential expression module analysis showed both ITGAE and CD69 were significantly increased in HPV+ when compared with HPVSCCs. (B) Kaplan-Meier survival curves of patients with head and neck SCC showed that patients with increased ITGAE (CD103) and CD69 (red lines) had improved survival when compared with patients with lower levels of these genes (blue lines) in HPV+ SCCs (upper panels). In patients with HPV- SCCs, however, there were no significant differences in patient survival (bottom panels). Statistical significance computed by differential analysis (edgeR) on RNA-Seq raw count ${ }^{\star \star \star} p<0.001$. HPV, human papillomavirus; SCC, squamous cell carcinoma.

compared with HPV- HNSCCs (figure 1A). Within the HPV+ HNSCC group, a higher than the median expression levels of CD103 or CD69 were associated with significantly better overall survival (figure 1B). By comparison, there was no correlation between the level of CD103 or CD69 expression and patient survival with HPV- HNSCCs (figure 1B). These data suggest that HPV+ HNSCCs may be characterized by increased tumor-resident CD8+ T cells and that the numbers of these $\mathrm{T}$ cells may be prognostic in HPV+ HNSCCs, but not in HPV- HNSCCs.

\section{HPV+ OPSCC has significantly higher proportion of CD103+CD8+ tumor-resident T cells compared with HPV- OPSCC}

The baseline characteristics by HPV status of the study cohort are shown in online supplementary table 1 . Of 62 patients, 35 (56.5\%) had HPV+ OPSCC. These patients were more likely to be never smokers $(48.6 \%)$, and had higher tumor grade $(54.3 \%$ grade 3$)$, higher $\mathrm{N}$-stage $(59.3 \% \mathrm{~N} 2 / \mathrm{N} 3)$ and higher programmed death-ligand 1 (PD-L1) expression (94.3\%), compared with patients with HPV- OPSCC. Patients with HPV+ OPSCCs had a significantly better overall survival than those with HPV- OPSCCs (figure 2A). The median overall survival of patients with HPV- OPSCC was 62.2 months, and the median survival for patients with HPV+ OPSCC was not reached. There was a significant difference in the 5 -year overall survival between the two groups $(82.3 \%$ for patients with HPV+ OPSCC vs $51.9 \%$ for patients with HPVOPSCC, $\mathrm{p}=0.014$ ). Multiplex quantitative IHC staining of tumor biopsy specimen showed increased numbers of CD8+ T cells in HPV+ OPSCCs when compared with HPV- OPSCCs, although the difference did not reach significance (figure 2B,C). CD103 staining showed that there was significantly more CD103+CD8+ T cells in HPV+ OPSCC than HPV- OPSCC $(4.8 \%$ vs $1.06 \%$ of all nucleated cells, $\mathrm{p}=0.0001$ ) (figure 2B,D). There was no significant difference in CD103-CD8+ T cell numbers between the HPV+ and HPV- OPSCC (figure 2E). Together these data demonstrate that HPV+ OPSCCs have significantly more $\mathrm{CD} 103+\mathrm{CD} 8+$ Trm $\mathrm{T}$ cells when compared with HPV- OPSCCs.

\section{Intratumoral CD103+CD8+ T cells are associated with best prognosis in HPV+ OPSCCs}

The location of $T$ cells can be a critical factor in influencing the effectiveness of tumor control. ${ }^{1123}$ We reasoned that persistent viral infection may have impacted on the intratumoral accumulation of CD103+CD8+ T cells in HPV+ OPSCCs. The specimens were segmented into tumor and 

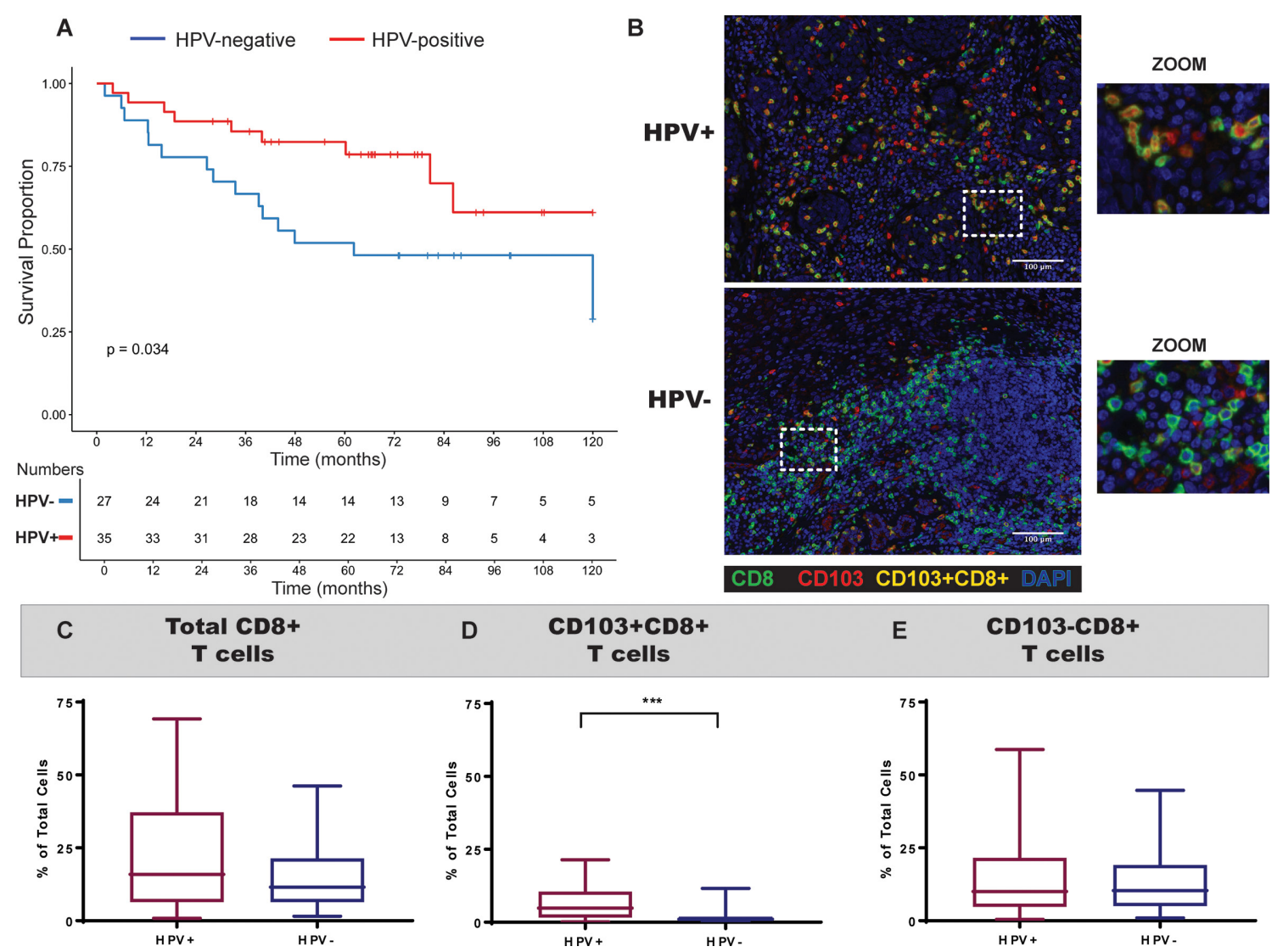

Figure 2 Significantly more CD103+CD8+ T cells in HPV+ OPSCCs when compared with HPV- OPSCCs. (A) KaplanMeier survival curves of patients with OPSCC from the study cohort with HPV+ (red; $n=36$ ) and HPV- (blue; $n=26)$ OPSCCs showed better overall survival for patients with HPV+ OPSCCs. (B) When tumor biopsy specimen was stained for CD103, CD8 and nucleus marker DAPI by multiplex IHC staining, it showed increased numbers of tumor-resident CD8+ T cells in HPV+ OPSCCs when compared with HPV- OPSCCs. (C-E) When the proportion of all nucleated cells expressing these markers was quantified as total CD8+ T cell population (C), CD103+CD8+ population (D) and CD103-CD8+ Tcell population (E), it was clear that CD103+CD8+ T cells were significantly enhanced in HPV+ OPSCCs when compared with HPV- OPSCCs. Statistical differences were calculated using unpaired and non-parametric Mann-Whitney t-test (** $p<0.001$; ns $p>0.05)$. HPV, human papillomavirus; IHC, immunohistochemistry; OPSCC, oropharyngeal squamous cell carcinoma.

stroma regions and then enumerated the $\mathrm{CD} 103+$ and CD103- CD8+ T cells. Both CD103+ and CD103- CD8+ $\mathrm{T}$ cells were present in both regions, and there were no significant differences between the regions in the overall study cohort (figure 3A-D). However, HPV+ OPSCG had a significantly higher measurement of both intratumoral $(2.8 \%$ vs $0.42 \%$ of all nucleated cells, $\mathrm{p}<0.0001)$ and stromal $(1.57 \%$ vs $0.68 \%, \mathrm{p}<0.005)$ CD103+CD8+ Tcell numbers when compared with HPV- OPSCG (figure 3A,B). By contrast, the proportions of CD103$\mathrm{CD} 8+\mathrm{T}$ cells were not significantly different between $\mathrm{HPV}+$ and HPV- OPSCC at either region (figure 3C,D). When we examined the cell distribution between each tumor type, CD103+CD8+ T cells were equally distributed between tumor and stroma in both HPV+ and HPVOPSCCs (online supplementary figure 1). CD103-CD8+ $\mathrm{T}$ cells accumulated significantly more in the stroma in both HPV+ and HPV- OPSCCs (online supplementary figure 1).

We then determined the impact of CD103+CD8+ T cell numbers and their location on survival in patietns with HPV+ OPSCCs. Kaplan-Meier plots showed that patients with higher intratumoral CD103+CD8+ T cells had significantly better survival when compared with patients with lower numbers $(\mathrm{p}=0.0078$; figure $3 \mathrm{E})$. The 5-year survival was $83.6 \%$ vs $54 \%(p=0.018)$. The amount of stromal CD103+CD8+ $\mathrm{T}$ cells can also influence the overall survival. The 5-year survival for those with high stromal CD103+CD8+ T cells was $83.4 \%$ compared with $53.9 \%$ for those with low stromal CD103+CD8+ T cells (figure 3F). By contrast, while there was a trend toward better survival with increased intratumoral CD103-CD8+ cells $(\mathrm{p}=0.053$; figure $3 \mathrm{G}$ ), their numbers in the stroma were not associated with any survival advantage (figure $3 \mathrm{H}$ ). Together, these data demonstrate that $\mathrm{CD} 103+\mathrm{CD} 8+\mathrm{T}$ cells were equally distributed between tumor and stroma, and increased numbers at both sites were associated with enhanced survival in patient with HPV+ OPSCC.

\section{CD103+CD8+ T cell numbers rather than HPV status underlie survival advantage in HPV+ OPSCCs}

The effects of a combination of HPV status and Trm level on outcomes are shown in multivariate Kaplan-Meier models (figure 4A,B). The study cohort was grouped by 


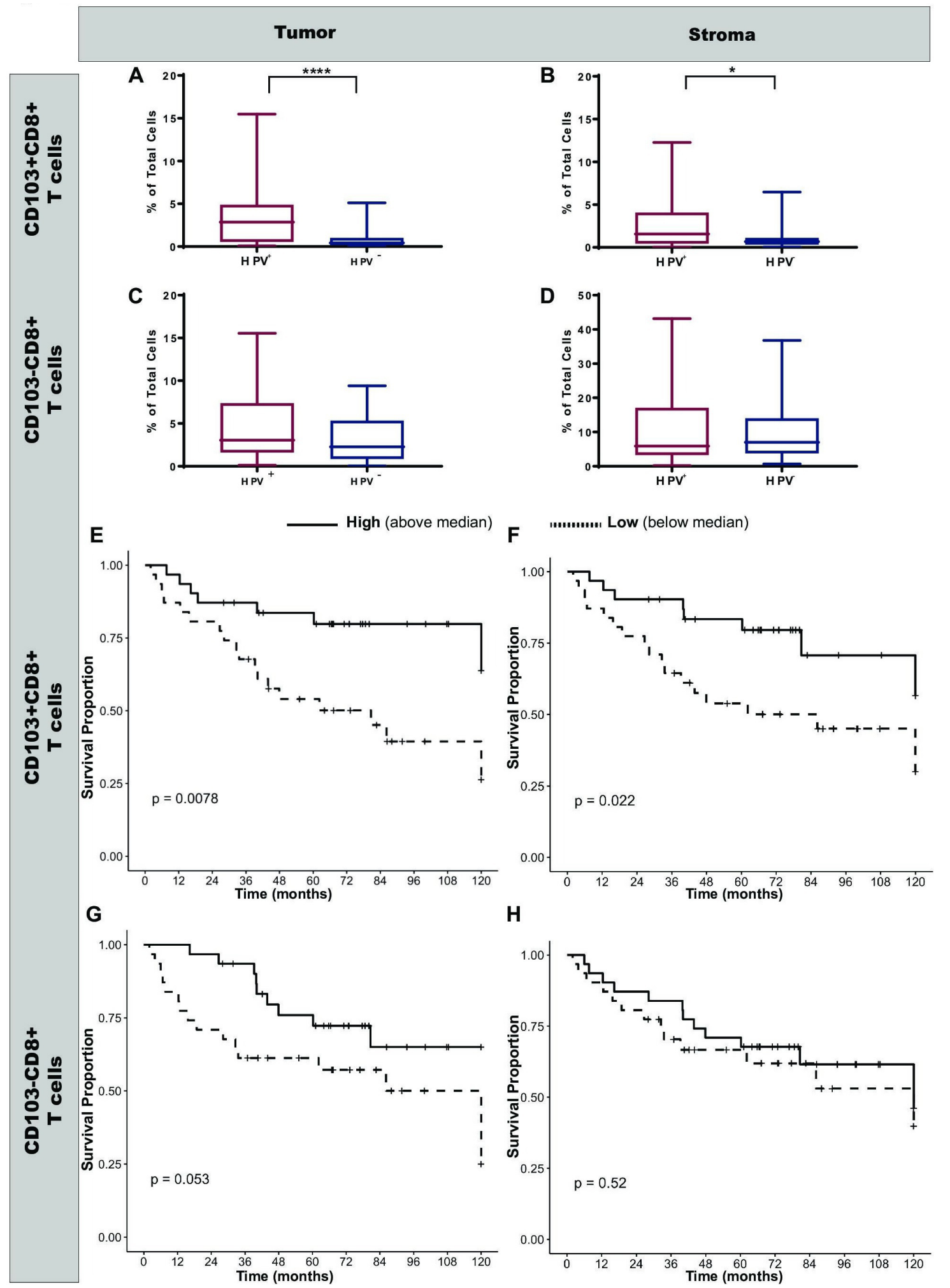

Figure 3 Enhanced intratumoral CD103+CD8+ Tcell numbers are associated with best patient survival. Tumor tissues examined by IHC stains were segmented into intratumoral regions and stromal regions, and the distribution of CD8+ Tcell subsets was quantified. CD103+CD8+ Tcell numbers in tumor regions (A) and stromal regions (B) showed that significantly more CD103+CD8+ T cells were present in both these regions in HPV+ OPSCCs when compared with HPV- OPSCCs. By contrast there were no significant differences in the CD103-CD8+ T cell numbers in those regions between HPV+ and HPVOPSCCs (C and D). Data are expressed as box and whisker plots and statistical differences were calculated using unpaired and non-parametric Mann-Whitney t-test. $(\mathrm{E}-\mathrm{H})$ Kaplan-Meier plots show the overall patient survival based on intratumoral $(\mathrm{E}$ and G) or stromal $(F$ and $H)$ distribution of CD103+CD8+ (E and F) and CD103-CD8+ (G and H) cells in HPV+ OPSCC. Statistical differences were calculated using unpaired and non-parametric Mann-Whitney t-test $\left(^{\star \star \star *} p<0.0001 ;{ }^{*} p<0.05 ; n s p>0.05\right)$. HPV, human papillomavirus; IHC, immunohistochemistry; OPSCC, oropharyngeal squamous cell carcinoma. 

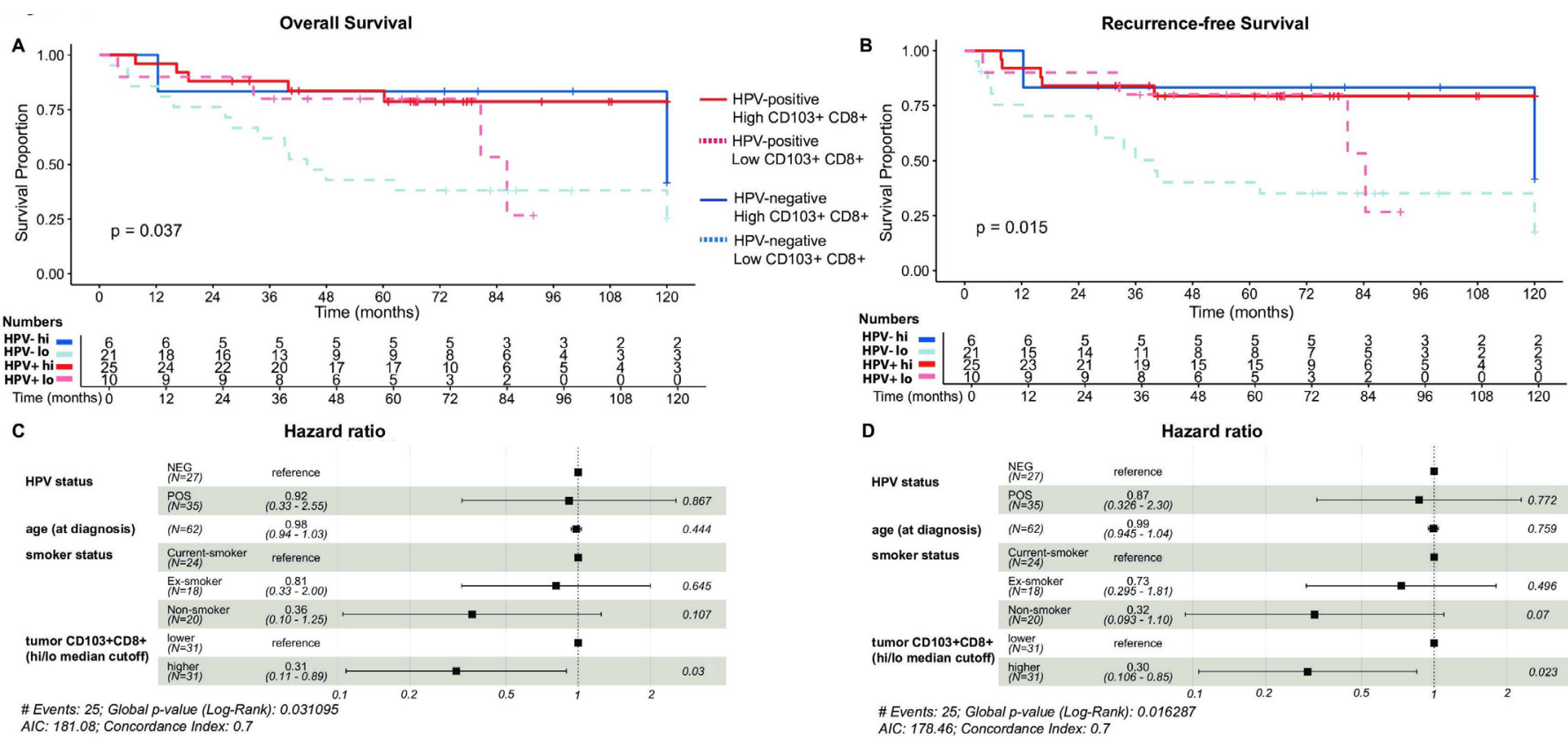

Figure 4 Increased CD103+CD8+ Tcell numbers are associated with improved survival in both HPV+ and HPV- OPSCCs. Kaplan-Meier plots for overall survival (A) or recurrence-free survival (B) for patients with OPSCCs based on their HPV status (HPV+, red lines; HPV-, blue lines) and the number of intratumoral CD103+CD8+ T cells show patients with increased CD103+CD8+ T cells had the best survival in both HPV+ and HPV- OPSCCs. The threshold for CD103+CD8+ cell numbers was set based on the median value of the overall cohort (high: solid lines; low: broken lines). Multivariate analysis shows intratumoral CD103+CD8+ Tcell numbers were the key differentiator between patients with HPV+ OPSCCs and HPV- OPSCCs for overall survival (C) and recurrence-free survival (D). Statistical differences were calculated using univariate analysis (Cox proportional Hazards model) ("$\left.{ }^{*}<0.05\right)$. HPV, human papillomavirus; NEG, negative; OPSCC, oropharyngeal squamous cell carcinoma; POS, positive; AIC, Akaike information criterion.

HPV status and Trm cell level (high vs low) (supplementary figure 2). Patients with HPV+ OPSCC had favorable survival regardless of the Trm cell level. Notably, patients with HPV- OPSCC and high level of CD103+CD8+ T cells had similar overall survival (figure $4 \mathrm{~A}$ ) and recurrencefree survival (figure 4B) to those patients with HPV+ OPSCC. The worst survival was seen in patients with HPV- OPSCC and low Trm level. In the multivariate analysis, intratumoral Trm level was a significant predictor of overall survival (HR $0.31,95 \%$ CI 0.11 to $0.89, p=0.03$; figure $4 \mathrm{C}$ ) and recurrence-free survival (HR $0.3,95 \% \mathrm{CI}$ 0.106 to $0.85, p=0.023$; figure $4 \mathrm{D}$ ). These data strongly suggest that CD103+CD8+ Tcell numbers rather than HPV status impact the survival of patients with OPSCCs.

\section{CONCLUSIONS}

Recently the American Joint Committee on Cancer has included HPV status in the staging system of OPSCC $^{24}$ as it is a strong prognostic factor in addition to $\mathrm{T}$ stage, $\mathrm{N}$ stage and $\mathrm{M}$ stage classification. Immunotherapy is also being increasingly used in the treatment of OPSCC..$^{25}$ A greater understanding of immune landscape of OPSCCcan help to develop novel prevention and treatment strategies. Here we show that the underlying mechanism for the better survival seen in HPV+ OPSCC is largely mediated by local $\mathrm{T}$ cell immunity provided by enhanced CD103+CD8+ Trm T cells. HPV+ OPSCC have higher level of Trm T cells than HPV- OPSCC. Importantly, our data also show that patients with HPV- OPSCC showing a high level of CD103+CD8+ Trm T cells in the tumor region had comparable survival with those patients with HPV+ tumors. This suggests that the critical determinant of survival is the effectiveness of the immune response rather than the virus status.

HPV is thought to infect the basal cells of the tonsillar crypt epithelium. ${ }^{26}$ As an immune population adapted to providing protection at such barrier surfaces, CD103+CD8+ T cells are likely to localize near the epithelium to prevent viral replication. These cells are also likely to express CD69, another marker of tissue retention. Our previous work on tonsillar memory $\mathrm{T}$ cells indeed showed that $\mathrm{CD} 103+\mathrm{CD} 8+\mathrm{T}$ cells preferentially localized near the epithelium, particularly areas near the crypts. ${ }^{27}$ Accumulation of these cells could be critical for immune-mediated control mechanisms. In addition, another previous study examining CD4+ $\mathrm{T}$ cells in head and neck cancers also found CD69+CD4+ Tcell numbers to be significantly associated with better survival and better locoregional control. ${ }^{28}$ Interestingly, CD103+CD8+ T cells infiltration can occur in HPV- OPSCCs, and sufficient numbers can also provide survival advantage to patients, further supporting the role of tumor-resident $\mathrm{T}$ cells in tumor immunity. Our findings of better survival associated with enhanced Trm $\mathrm{T}$ cells could also be related to better 
response to radiation therapy. Radiation therapy is one of the main treatment modalities for OPSCC, and a recent study showed that local tumor $\mathrm{T}$ cell status contributes to response to radiotherapy. ${ }^{29}$ Pre-existing tumor $\mathrm{T}$ cells can survive radiation and mediate antitumor responses without the assistance from newly infiltrating T cells. ${ }^{29}$

Together our study shows that local $\mathrm{T}$ cell immunity provided by Trm $\mathrm{T}$ cells could be a critical determinant in providing favorable clinical outcomes in patients with OPSCCs. Therefore, strategies aiming to boost these Trm $\mathrm{T}$ cells at sites of tumor development could be beneficial.

\section{Author affiliations}

${ }^{1}$ Centenary Institute, Newtown, New South Wales, Australia

${ }^{2}$ Infectious Diseases and Immunology, School of Medical Sciences, Faculty of Medicine and Health, The University of Sydney, Sydney, New South Wales, Australia ${ }^{3}$ Charles Perkins Centre, The University of Sydney, Sydney, New South Wales, Australia

${ }^{4}$ School of Mathematics and Statistics, The University of Sydney, Sydney, New South Wales, Australia

${ }^{5}$ Central Clinical School, Faculty of Medicine and Health, The University of Sydney, Sydney, New South Wales, Australia

${ }^{6}$ Department of Immunology and Microbiology, The University of Melbourne at the Peter Doherty Institute for Infection and Immunity, Melbourne, Victoria, Australia ${ }^{7}$ Department of Radiation Oncology, Chris 0'Brien Lifehouse, Sydney, New South Wales, Australia

\section{Twitter Angela Hong @AngelaHong and Umaimainthan Palendira @mainthan}

Acknowledgements We thank the Australian Centre for Microscopy \& Microanalysis at the University of Sydney for their assistance with this work.

Contributors UP and AH were involved in the design, intellectual oversight, supervision, prepared and approved the final manuscript. JY and KW contributed to the statistical analysis of the data. RH and AF performed the experiments, analyzed the data and wrote the manuscript. DJ, JE and MZ performed the experiments and helped analyze the data. WB and TG provided intellectual input and helped prepare the manuscript.

Funding This work was funded by a Cancer Council NSW grant (RG18-08) and a Bio-Connect grant from the University of Sydney.

Competing interests None declared.

Patient consent for publication Not required.

Ethics approval The study was approved by the Sydney Local Health District Human Ethics Committee (Protocol X12-0141).

Provenance and peer review Not commissioned; externally peer reviewed.

Data availability statement Data are available in a public, open access repository. The Cancer Genome Atlas data used in this study are available through the Tumor Immune Estimation Resource server.

Open access This is an open access article distributed in accordance with the Creative Commons Attribution Non Commercial (CC BY-NC 4.0) license, which permits others to distribute, remix, adapt, build upon this work non-commercially, and license their derivative works on different terms, provided the original work is properly cited, appropriate credit is given, any changes made indicated, and the use is non-commercial. See http://creativecommons.org/licenses/by-nc/4.0/.

\section{ORCID iD}

Umaimainthan Palendira http://orcid.org/0000-0002-1113-3306

\section{REFERENCES}

1 Chaturvedi AK, Zumsteg ZS. A snapshot of the evolving epidemiology of oropharynx cancers. Cancer 2018;124:2893-6.

2 Hong A, Lee CS, Jones D, et al. Rising prevalence of human papillomavirus-related oropharyngeal cancer in Australia over the last 2 decades. Head Neck 2016;38:743-50.
3 Prue G, Baker P, Graham D, et al. It is time for universal HPV vaccination. Lancet 2018;392:913-4.

4 Pan C, Issaeva N, Yarbrough WG. HPV-driven oropharyngeal cancer: current knowledge of molecular biology and mechanisms of carcinogenesis. Cancers Head Neck 2018;3:1-11.

5 fan WH, sha WS, Tang YJ, et al. The double-edged sword-how human papillomaviruses interact with immunity in head and neck cancer. Front Immunol 2019;10:1-12.

6 Hong AM, Dobbins TA, Lee CS, et al. Use of cyclin D1 in conjunction with human papillomavirus status to predict outcome in oropharyngeal cancer. Int J Cancer 2011;128:1532-45.

7 Hong AM, Martin A, Armstrong BK, et al. Human papillomavirus modifies the prognostic significance of $\mathrm{T}$ stage and possibly $\mathrm{N}$ stage in tonsillar cancer. Ann Oncol 2013;24:215-9.

8 Stanley KK, Szewczuk E. Multiplexed tandem PCR: gene profiling from small amounts of RNA using SYBR green detection. Nucleic Acids Res 2005;33:e180-9.

9 Hong A, Jones D, Chatfield M, et al. HPV status of oropharyngeal cancer by combination HPV DNA/p16 testing: biological relevance of discordant results. Ann Surg Oncol 2013;20(Suppl 3):450-8.

10 Hong A, Zhang X, Jones D, et al. Relationships between p53 mutation, HPV status and outcome in oropharyngeal squamous cell carcinoma. Radiother Oncol 2016;118:342-9.

11 Park SL, Gebhardt T, Mackay LK. Tissue-Resident memory T cells in cancer immunosurveillance. Trends Immunol 2019;40:735-47.

12 Gebhardt T, Palendira U, Tscharke DC, et al. Tissue-resident memory $T$ cells in tissue homeostasis, persistent infection, and cancer surveillance. Immunol Rev 2018;283:54-76.

13 Edwards J, Wilmott JS, Madore J, et al. CD $103^{+}$tumor-resident CD8 T cells are associated with improved survival in immunotherapynaïve melanoma patients and expand significantly during anti-PD-1 treatment. Clin Cancer Res 2018;24:3036-45.

14 Komdeur FL, Prins TM, van de Wall S, et al. CD103+ tumorinfiltrating lymphocytes are tumor-reactive intraepithelial CD8+ T cells associated with prognostic benefit and therapy response in cervical cancer. Oncoimmunology 2017;6:e1338230-14.

15 Webb JR, Milne K, Watson P, et al. Tumor-infiltrating lymphocytes expressing the tissue resident memory marker cd103 are associated with increased survival in high-grade serous ovarian cancer. Clin Cancer Res 2014;20:434-44.

16 Savas P, Virassamy B, Ye C, et al. Single-cell profiling of breast cancer $T$ cells reveals a tissue-resident memory subset associated with improved prognosis. Nat Med 2018;24:986-93.

17 Djenidi F, Adam J, Goubar A, et al. CD8+CD103+ tumor-infiltrating lymphocytes are tumor-specific tissue-resident memory $T$ cells and a prognostic factor for survival in lung cancer patients. J Immunol 2015;194:3475-86.

18 Ganesan A-P, Clarke J, Wood O, et al. Tissue-resident memory features are linked to the magnitude of cytotoxic $T$ cell responses in human lung cancer. Nat Immunol 2017;18:940-50.

19 Park SL, Buzzai A, Rautela J, et al. Tissue-resident memory CD8 ${ }^{+}$ T cells promote melanoma-immune equilibrium in skin. Nature 2019;565:366-71.

20 Weinberger PM, Yu Z, Haffty BG, et al. Molecular classification identifies a subset of human papillomavirus--associated oropharyngeal cancers with favorable prognosis. J Clin Oncol 2006;24:736-47.

21 Smeets SJ, Hesselink AT, Speel E-JM, et al. A novel algorithm for reliable detection of human papillomavirus in paraffin embedded head and neck cancer specimen. Int J Cancer 2007;121:2465-72.

22 Li T, Fan J, Wang B, et al. TIMER: a web server for comprehensive analysis of tumor-infiltrating immune cells. Cancer Res 2017;77:e108-10.

23 Oguejiofor K, Hall J, Slater C, et al. Stromal infiltration of CD8 T cells is associated with improved clinical outcome in HPV-positive oropharyngeal squamous carcinoma. Br J Cancer 2015;113:886-93.

24 O'Sullivan B, Huang SH, Su J, et al. Development and validation of a staging system for HPV-related oropharyngeal cancer by the International collaboration on oropharyngeal cancer network for staging (ICON-S): a multicentre cohort study. Lancet Oncol 2016;17:440-51.

25 Gillison ML, Blumenschein G, Fayette J, et al. CheckMate 141: 1 -year update and subgroup analysis of nivolumab as first-line therapy in patients with recurrent/metastatic head and neck cancer. Oncologist 2018;23:1079-82.

26 Egawa N, Egawa K, Griffin H, et al. Human papillomaviruses; epithelial tropisms, and the development of neoplasia. Viruses 2015;7:3863-90.

27 Woon HG, Braun A, Li J, et al. Compartmentalization of total and virus-specific tissue-resident memory CD8+ T cells in human lymphoid organs. PLoS Pathog 2016;12:e1005799-19. 
28 Badoual C, Hans S, Rodriguez J, et al. Prognostic value of tumorinfiltrating CD4+ T-cell subpopulations in head and neck cancers. Clin Cancer Res 2006;12:465-72.
29 Arina A, Beckett M, Fernandez C, et al. Tumor-reprogrammed resident $\mathrm{T}$ cells resist radiation to control tumors. Nat Commun $2019 ; 10$. 